CAHIERS DE

NARRATOLOGIE

\section{Cahiers de Narratologie}

Analyse et théorie narratives

$28 \mid 2015$

Le récit comme acte cognitif

\title{
Forces et espaces : Maupassant, Borges, Hewingway
}

Éléments de narratologie sémio-cognitive*

Per Aage Brandt

\section{OpenEdition}

Journals

Electronic version

URL: https://journals.openedition.org/narratologie/7291

DOI: 10.4000/narratologie.7291

ISSN: 1765-307X

Publisher

LIRCES

Electronic reference

Per Aage Brandt, "Forces et espaces : Maupassant, Borges, Hewingway", Cahiers de Narratologie

[Online], 28 | 2015, Online since 29 October 2015, connection on 11 June 2021. URL: http://

journals.openedition.org/narratologie/7291; DOI: https://doi.org/10.4000/narratologie.7291

This text was automatically generated on 11 June 2021.

Article L.111-1 du Code de la propriété intellectuelle. 


\section{Forces et espaces : Maupassant, Borges, Hewingway}

Éléments de narratologie sémio-cognitive*

Per Aage Brandt

\section{AUTHOR'S NOTE}

*Le terme de « narratologie » a été forgé par Todorov (1969 : 10). L'expression " narratologie cognitive » est beaucoup plus récente et a été utilisée pour la première fois, me semble-t-il, dans le sens adopté par cet article, par David Herman (2000). Le terme de « semio-cognitif » renvoie à une approche récente, dans la recherche, qui s'appuie en particulier sur la sémiotique (structurale et philosophique) et sur la sémantique cognitive. Voir Cognitive Semiotics. Multidisciplinary Journal on Meaning and Mind (2007-) et Brandt (2004b).

\section{Discours narratif}

1 Si par «discours" nous entendons l'ensemble des propriétés sémantiques que les phrases doivent partager afin d'avoir un sens en tant que parties du flux de communication langagière, nous pouvons toutefois distinguer différents types ou genres dans ce flux discursif, présentant des propriétés sémantiques distinctes.

2 Il est ainsi possible de distinguer les discours narratif, descriptif et argumentatif ${ }^{1}$, dans la mesure où ces genres présentent des différences de base sémantiques. Le discours narratif déroule une continuité temporelle et spatiale ou, du moins, une certaine continuité en ce qui concerne les états, les faits et les événements, les objets et les personnages, alors que le discours descriptif présente des inventaires d'entités synchroniques qui se contentent de partager un espace spécifique ${ }^{2}$. Quant au discours argumentatif, il fait essentiellement dériver des représentations d'autres représentations, tant narratives que descriptives, en combinant les propriétés 
conceptuelles de leurs contenus. Il est en outre évident que les récits peuvent contenir des descriptions et des passages argumentatifs, qu'on peut argumenter au sujet de récits et de descriptions (c'est ce que la recherche historique fait) et que tout peut être décrit (toutefois, décrire une histoire, ce n'est pas la raconter, et décrire un argument, ce n'est pas argumenter).

3 Je voudrais souligner un autre trait structurel qui caractérise le discours narratif : c'est le seul type de discours qui présente une dualité sémantique constitutive dans les représentations de l'espace, à savoir une coupure entre la représentation perceptuelle et conceptuelle du même espace d'événements, et la projection de l'une sur l'autre. En d'autres termes, une coupure entre le temps-espace proche, "concret» et phénoménologique du personnage impliqué et le même temps-espace du narrateur, dans sa version distante et abstraite, fondée sur un savoir ou un non-savoir. Par opposition, le discours purement argumentatif n'est en principe que conceptuel et distant ou abstrait, alors que le discours purement descriptif n'est que perceptuel et proche ou concret. Cette double représentation de l'espace-temps narratif se manifeste dans des genres narratifs pragmatiques tels que les comptes-rendus de navigation (les rapports de voyage, par exemple) et de guerre (incluant les plans stratégiques), dans lesquels les cartes sont un moyen central de référence. Être "sur" une carte, c'est intégrer une deixis perceptuelle et une géographie conceptuelle. La double représentation est particulièrement marquée dans la littérature narrative et constitutive de la fiction en général, puisque la perception fictive doit prendre place dans un contexte créé par la manipulation conceptuelle explicite et ludique d'un savoir commun ${ }^{3}$.

4 Toutes les manifestations du discours narratif, des entités sémiotiques que nous appelons histoires ${ }^{4}$, qu'elles soient fictives ou «factives » (c'est-à-dire documentaires) présentent un ensemble de " personnes » formant un noyau : des instances subjectives, incluant une première série de personnages impliqués (protagonistes ou observateur); ensuite, une série supplémentaire d'agents; enfin, la voix du narrateur (conceptuel) dominant en principe la chaîne des événements de l'histoire, mais souvent incarné dans une instance subjective (primaire ou supplémentaire), permettant ainsi un « point de vue ${ }^{5}$ » spécifique. Ces instances qui incluent la voix narrative de base elle-même, se déploient uniquement dans la mesure où elles participent et assistent à la même chaîne d'événements, c'est-à-dire à l'histoire en tant que telle. Il doit y avoir une Histoire en soi, pour ainsi dire ${ }^{6}$. Ce principe d'une distinction entre la narration (fondée sur la voix) et l'histoire (fondée sur les événements) est, je crois, une présupposition cognitive applicable à tous les discours narratifs, une condition propre au fait d'avoir un sens, une condition de sa possibilité cognitive et sémiotique, et il représente, par conséquent, le point de départ naturel d'une narratologie sémio-cognitive ${ }^{7}$.

5 Les événements sont liés dans une chaîne de faits qui constitue une histoire, dans la mesure où ils se produisent dans un continuum temporel formant une trajectoire dans un continuum spatial et où certains de ces événements sont critiques. Un événement critique est un changement d'état "significatif ", qui survient à un endroit ou à un autre dans ce continuum spatio-temporel ${ }^{8}$. Je vais tenter de montrer qu'il est le résultat de forces créant des états dans des espaces. Un changement critique est le résultat d'une variation de l'ampleur relative de certaines forces stabilisantes, qui s'affrontent et s'opposent localement, ce qui entraîne d'autres variations de forces locales. L'intérêt de cette vision dynamique est que les espaces représentés, en tant que champs opératifs 
limités de forces stabilisant et changeant des états, doivent être « locaux » et articulés, chaque "localité" contenant une disposition qui encadre un déploiement caractéristique de forces".

6 Il semblerait par conséquent pertinent sur le plan phénoménologique d'étudier et d'élaborer une typologie représentative de force ${ }^{10}$ se produisant dans des expériences connues, partagées par des êtres humains, dans ce que les philosophes appellent notre "monde vécu », Lebenswelt. Ces forces constitueraient l'inventaire cognitif naturel des forces qui ont plus de chances de surgir dans des espaces narratifs, qu'elles soient littéraires, religieuses ou bien culturelles (par exemple, mythologiques). Nous allons maintenant nous risquer à proposer ici une courte liste de types immédiatement perceptibles, supposés actifs dans la dynamique de la vie quotidienne ${ }^{11}$. Il y a les forces intentionnelles, potentiellement conscientes, qui, par "causalité mentale ", motivent les actes de sujets incarnés. Ces derniers les "contiennent" comme forces agentives, à moins que les sujets ne les rencontrent et ne les manipulent en tant que forces magiques de la nature. Les forces agentives et magiques sont toutes deux intentionnelles, au sens où la volition est impliquée, de sorte que la magie est facilement réincarnée dans les fées, les trolls, les fantômes et les divinités de tout genre (mais il n'est pas nécessaire qu'elle soit incarnée). Il y a en outre, les forces causales " aveugles ", sur lesquelles ces sujets comptent et qu'ils rencontrent dans les domaines physiques et sociaux. Certaines d'entre elles sont banales, c'est-à-dire habituelles, attendues, inhérentes à l'état des choses et quasi nécessaires dans un espace circonstanciel donné, alors que d'autres, à l'opposé, ne sont pas prévisibles: contingentes, possibles mais rares, soudaines, ponctuelles, singulières et localement fatales, à savoir, en dépit de leur singularité et de leur caractère ponctuel, décisives pour le cours narratif des événements. Elles sont significatives et critiques ${ }^{12}$.

7 Je voudrais montrer qu'il est possible de comprendre de manière significative la logique événementielle des histoires en ces termes d'interaction de forces, uniquement ou essentiellement, et par là de cerner les structures centrales de la fabrication du sens des récits de base que toute narration présuppose, qu'elle soit artistique ou non. La forme artistique, littéraire, provient souvent d'une structure narrative épisodique quotidienne. Elle apporte des explications supplémentaires descriptives et argumentatives à des expériences supposées subjectives, elle décrit ou discute sa propre manière de narrer. En général, la logique événementielle reste la même.

\section{Genres narratifs}

8 Afin de comprendre et d'évaluer une histoire, nous décidons souvent implicitement à quel genre elle appartient. Dans la sémiotique des histoires, les genres sont l'équivalent des espèces en biologie - nous les appliquons pour obtenir une catégorisation rapide et utile. Toutefois, nous avons ici affaire à l'imaginaire humain, et ses représentations sémantiques suivent le format sémio-cognitif des objets imaginés, quel que soit leur degré de "naturalisme» ou de "créativité ». Les genres narratifs se distinguent spontanément grâce à leurs propriétés dynamiques. Ainsi, une "bonne » légende hagiographique et un "bon » témoignage donné par un témoin à un procès, suivant tous deux un format narratif parfait, sont rarement « bons » ou bien composés selon les mêmes critères. Le premier présente souvent des forces plus fatales et plus magiques que le second ${ }^{13}$. Si nous percevons qu'il existe plusieurs genres narratifs, dont relèvent 
les histoires fantastiques versus les histoires socio-réalistes, les histoires grotesques ou absurdes versus les contes de fées et ce, en raison de leurs styles dynamiques, c'est-àdire de leur admission ou non-admission de tel ou tel type d'événements spécifiques (soumis à des forces), nous pourrons développer ce point de vue en concevant une séquence totalement narrative d'espaces liés temporellement et connectés dynamiquement, en tant que configuration d'un monde ou en tant que manifestation d'un type de monde narratif spécifique dynamiquement. Dans certains mondes narratifs, les forces intentionnelles dominent et la magie est la bienvenue, alors que le reste relève d'une causalité banale. Dans d'autres mondes, c'est la causalité non-banale, historique et fatale qui domine. Et ainsi de suite... Les propriétés dynamiques des genres narratifs distincts diffèrent apparemment en raison de la nature des mondes conceptuels dont leurs espaces dynamiques relèvent. La sonorité et la température (froide ou chaude) produites par la voix du narrateur "olympien» non incarné peuvent alors être perçues comme le mode d'expression d'un monde narratif conceptuel donné.

9 Ce qui suit est l'ébauche élémentaire d'une telle présentation des genres narratifs, en termes de « mondes».

10 Nous pouvons présupposer une base d'ancrage neutre et naturaliste, un monde $\mathrm{R}$ tout simplement réaliste, correspondant au domaine de la vie quotidienne et de la cognition communément humaines, macro-physiques, mentales, sociales et pragmatiques. $\mathrm{Ce}$ monde de base réaliste peut être narrativement déformé de deux manières, par l'addition ou par la soustraction de forces ${ }^{14}$. Nous ajoutons des forces narratives, si nous permettons à des faits magiques de se produire en même temps que des causes réalistes dans un monde donné. Cela augmente son intensité dynamique et le résultat en est soit le genre fantastique (dans lequel la magie est locale), soit le genre enchanté, merveilleux (dans lequel la magie est globale ${ }^{15}$ ). En revanche, si nous retirons des forces, nous permettons que se produisent des événements et des faits qui n'ont aucune raison banale, causale ou intentionnelle. Cela diminue l'intensité dynamique de l'histoire et nous obtenons un monde plus «fou ». Ce fonctionnement entraîne les genres grotesque ou absurde. Les forces banales et fatales sont là abandonnées à elles-mêmes sur la scène, alors que les forces intentionnelles diminuent. Cette conjoncture provoque souvent de l'humour ou une impression de «folie » ou encore des situations et des comportements incompréhensibles (on peut penser au Rhinocéros d'Eugène Ionesco, par exemple). Le réalisme narratif peut être ainsi déformé dans deux directions opposées, soit vers le fantastique et le merveilleux, à l'intensité dynamique augmentée, soit vers le grotesque et l'absurde (à l'intensité dynamique diminuée). Le magique est intuitivement une forme marquée (au sens défini par Roman Jakobson) de force intentionnelle (alors que l'intentionnalité agentive n'est pas marquée, elle existe par défaut). Quant à la force fatale, causale, elle est par conséquent marquée (alors que la causalité banale est bien entendu non marquée). C'est la raison pour laquelle le réalisme est le genre le moins marqué. Le merveilleux et l'absurde sont en revanche les plus marqués.

11 La question du statut des genres en littérature est bien évidemment beaucoup plus complexe que nous le discutons ici. Les genres narratifs ne sont que des sous-genres $d u$ genre narratif en tant que tel et pourraient être pris pour des subdivisions arbitraires de ce dernier. Mais il y a de bonnes raisons pour penser que les spécifications dynamiques sont vraiment efficaces dans l'organisation cognitive humaine du sens, de telle sorte qu'elles "génèrent» du sens différemment. Elles sont, en fait, organiquement 
distinctes. La spécification psychologique des personnages, par exemple, est de moins en moins possible à mesure que la magie augmente ou que la fatalité augmente. La longueur même des histoires a également tendance à diminuer pour des raisons identiques: les contes de fées et les histoires absurdes sont habituellement courts. Seulement des références cognitives peuvent expliquer de telles corrélations, me semble-t-il. L'émerveillement magique et l'humour absurde sont des processus de courte durée dans l'esprit humain.

12 Le schéma suivant est une tentative légèrement simplifiée de résumer cette vision des genres narratifs (les mondes sont entre parenthèses) :

Fig. 1

\section{Les genres et leurs mondes narratifs}

\section{MERVEILLEUX FANTASTIQUE RÉALISTE GROTESQUE ABSURDE}

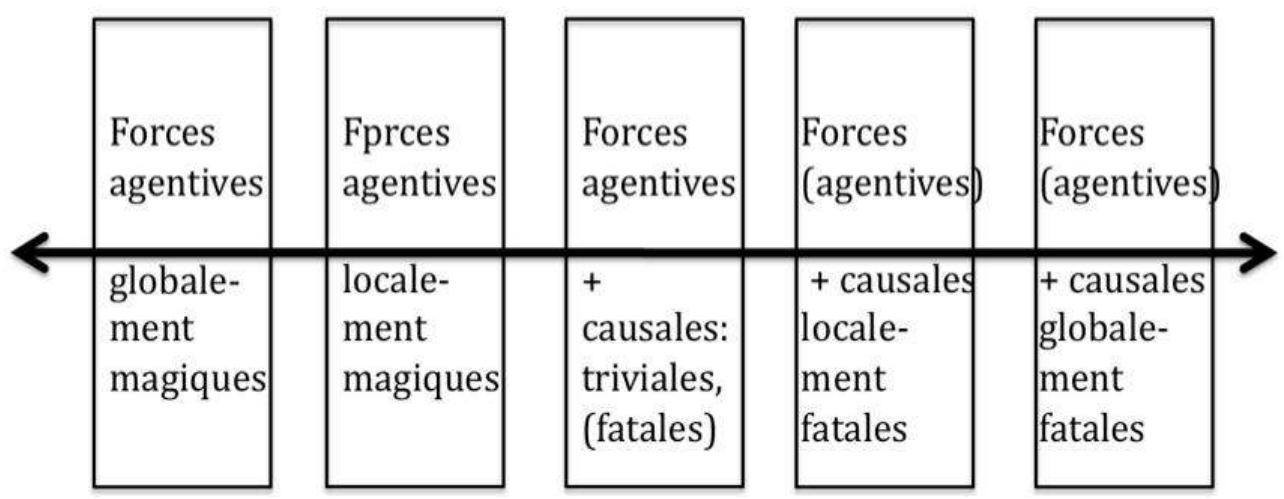

Dans cette vision des choses, un scénario narratif, un épisode, une séquence d'événements et d'actes, toute unité diégétique est une partie du "monde » caractérisé par un «mélange » spécifique de propriétés dynamiques, réalistes ou déformées dans une direction ou une autre. Certaines histoires peuvent même changer de « monde " au cours des longs développements comportant de nombreux espaces de scénarios différents. Pensons, par exemple, à Cent ans de solitude de Gabriel García Márquez ou à Alice au pays des merveilles et à De l'autre côté du miroir de Lewis Carroll, qui oscillent radicalement sur l'axe des "mondes", entre le merveilleux et l'absurde ${ }^{16}$. Les auteurs ont des préférences en matière de "mondes", qui sont souvent leur signature - Kafka : le grotesque; Kharms, Ionesco: l'absurde; Cortázar, Borges, Poe: le fantastique; Breton, Aymé : le merveilleux. Cela relève probablement du profil cognitif général des écrivains. Cela dit, un auteur n'est évidemment pas enfermé dans un seul genre.

Les sous-genres narratifs sont importants pour l'interprétation des histoires, parce qu'ils déterminent la manière dont les récits ont un sens pour nous: une histoire grotesque ou fantastique ne nous fait pas croire que la vie est grotesque ou fantastique. Elle peut nous faire rire ou trembler, pendant que nous retirons de son monde un sens pertinent pour notre vie, monde que nous distinguons du nôtre. Nos esprits cognitifs prennent essentiellement plaisir à visiter des mondes non-réalistes, marqués. Paradoxalement, de tels mondes sont ressentis comme porteurs de davantage de sens que le monde banal, non marqué. Il est clair que nos esprits émotionnels préfèrent les premiers. 
C'était le premier point important de cet article. Le second est l'hypothèse suivante : dans chaque histoire, un monde narratif est articulé en termes de séquences d'espaces événementiels, c'est-à-dire de lieux plus ou moins clairement définis, mis en place par des indications locatives ou par des descriptions, dans lesquelles les personnages sont reliés activement ou passivement au jeu des forces de différents types, qui sont en conflit, et ils sont affectés par ces forces à travers le temps narré. L'interconnexion d'espaces et de forces, nous permettant de détecter une série diégétique canonique de positions spatio-temporelles dynamiques à travers lesquelles une histoire prend son sens, est l'idée centrale de cette hypothèse. Il faut noter que les espaces événementiels ne sont pas des espaces mentaux. Un espace mental est la partie d'un réseau ancré dans une semiosis immédiate (voir Brandt 2004), alors qu'un espace événementiel est un cadre spatial de contenu narratif situé dans la diégèse d'une histoire. Une semiosis peut contenir une diégèse, dans la mesure où une narration contient un récit. Peut-être cela demandera une nouvelle initiative, celle de construire une théorie textuelle sémiotique et cognitive cohérente, pour approfondir cette relation et cette articulation. La sémantique des discours dans la prétendue Text World Theory, (Théorie des mondes textuels, Gavins 2007), pourrait offrir une contribution d'importance à un tel projet, bien qu'elle ne s'intéresse pas aux structures dynamiques immanentes de la causalité narrative, qui sont notre préoccupation principale dans ce texte. Est-il possible d'identifier un ensemble canonique d'espaces événementiels narratifs? Ce qui suit tente de répondre à cette question à travers l'analyse de textes littéraires.

16 Nous illustrerons notre approche en présentant une brève séquence de modèles spatiaux dynamiques, destinés à extraire le sens narratif de base de nouvelles de Guy de Maupassant (La parure, Deux amis, La ficelle), de Jorge Luis Borges (Emma Zunz, L'autre mort) et d'Ernest Hemingway (Une très courte histoire). Je voudrais souligner que dans une tentative d'analyse et de schématisation telle que celle-ci, les erreurs sont probables. Toutefois, l'avantage des schémas et des modèles explicites est que les erreurs sont plus faciles de relever et de discuter.

\section{Les dynamiques de forces spécifiques de la structure narrative}

1.

17 La parure ${ }^{17}$ de Guy de Maupassant est une histoire triste d'humiliation et de cruauté absurde du destin, dans laquelle un malentendu détruit une vie. Mme Loisel, qui vient d'une famille modeste mais rêve du luxe, est mariée à un pauvre employé du ministère de l'Instruction Publique. Lorsque son mari reçoit une invitation à un bal du ministère, elle parvient à obtenir de lui une nouvelle robe, mais elle estime avoir également besoin d'un bijou. Elle se rend, bien qu'à contrecœur, chez une amie riche, Mme Forestier, une ancienne camarade d'école, et lui demande de lui prêter un bijou. Parée d'une rivière de diamants, elle se rend au bal avec son mari et a beaucoup de succès. Le couple quitte la soirée à une heure tardive, en toute hâte, afin que leurs manteaux sans élégance n'attirent pas l'attention. C'est en arrivant à leur modeste demeure qu'ils découvrent que le collier a disparu. La semaine suivante, dans l'affolement, ils parviennent à trouver un collier identique qu'ils n'ont pas les moyens de payer. Ils se servent d'un héritage, empruntent une fortune et achètent la parure. Le couple passe 
les dix années suivantes à rembourser leur dette, vivant misérablement et travaillant jour et nuit, assurant plusieurs emplois. La jeunesse et la beauté de Mme Loisel se fanent. Et un jour, elle rencontre son ancienne amie, Mme Forestier, sur les ChampsÉlysées, toujours belle, se promenant avec un enfant. Elle lui raconte son histoire afin de lui expliquer pour quelle raison elle a changé à tel point que son amie ne l'a pas reconnue. C'est alors que Mme Forestier lui apprend que le collier n'était en fait qu'une imitation, de la verroterie sans valeur.

18 L'histoire met en place un espace initial, A, présentant les protagonistes chez eux et résumant les conditions précédant les événements qui vont se produire : la situation modeste du couple, les ambitions de la jeune femme, puis l'occasion qui s'offre à elle et les préparatifs pour le bal ${ }^{18}$. Le second espace, B, à savoir le scénario du bal ministériel, contient la métamorphose matérialisant le désir du protagoniste féminin et la catastrophe fatale qui met fin à cette brève transformation. Le troisième espace, $\mathrm{C}$, est un espace-temps de dix ans, évoquant les conséquences des états, des événements et des actes précédents. Enfin, le quatrième espace, $\mathrm{D}$, celui du boulevard parisien, contient la rencontre fortuite et la révélation du peu de prix de l'objet dont la perte a pourtant détruit la vie du couple.

Quatre espaces ou étapes s'articulent dans cette nouvelle ${ }^{19}$. Considérons maintenant l'inventaire dynamique et les connexions de forces.

20 A : L'espace-temps de la modeste vie petite-bourgeoise.

A1. La force intentionnelle agentive de Mathilde, son désir de luxe et de charme.

A2. La force causale banale de la condition sociale dont elle est issue et dans laquelle son mariage l'a maintenue.

A3. La force causale fatale de l'invitation au bal mondain.

A4. La force intentionnelle agentive des préparatifs pour le bal, incluant l'emprunt du collier à Mme Forestier. $\mathrm{A} 2 \rightarrow(\mathrm{A} 3 \rightarrow \mathrm{A} 4)$.

21 B : Le bal ministériel.

B1. La force causale banale de la joie immédiate et du charme du bal, faisant écho à A1.

B2. La force causale fatale de la perte du collier, soulignée par la continuation de A2.

C : L'espace-temps social, couvrant de nombreuses années de difficultés.

C1. La conséquence causale banale de B2, à savoir de lourdes dettes.

C2. La conséquence causale banale de $\mathrm{C} 1$, c'est-à-dire dix années irréversibles de dur labeur et de misère.

D. L'espace de la rencontre, les Champs Élysées.

D1. La conséquence causale banale de C2, l'état d'épuisement de Mathilde.

D2. La rencontre causale fatale de Mathilde et de celle qui lui avait prêté le collier (A4).

D3. La conséquence causale banale de D1, à savoir que Mme Forestier ne reconnaît pas Mathilde et que cette dernière doit lui raconter son histoire.

D4. La force causale fatale de la découverte de Mathilde, c'est-à-dire que B2 $\rightarrow \mathrm{C} 1$ était fondé sur un malentendu en raison de A2 + A1 (Mathilde avait honte de sa situation et refusait de voir Mme Forestier). Le contenu de D4, le malentendu, annule B2 $\rightarrow \mathrm{C} 1$, mais malheureusement après que $\mathrm{C} 1 \rightarrow \mathrm{C} 2$. C'est là un cas classique d'anagnorisis aristotélicienne ${ }^{20}$.

24 Nous appellerons ici A un espace de condition, B un espace de catastrophe, C un espace de conséquence et $\mathrm{D}$ un espace de conclusion. Ces espaces narratifs sont déployés comme des lieux d'expérience distincts. C'est nettement le cas ici pour $B$ et $D$, alors que $A$ et $C$ sont 
décrits de manière plus vague. Ces derniers sont toutefois clairement distincts de leurs espaces voisins. Le réseau spatio-causal dans sa totalité peut être synthétisé et visualisé (fig. $2^{21}$.

Fig. 2. Réseau spatio-dynamique de La parure

\section{A : CONDITION B : CATASTROPHE C: CONSÉQUENCE D : CONCLUSION}

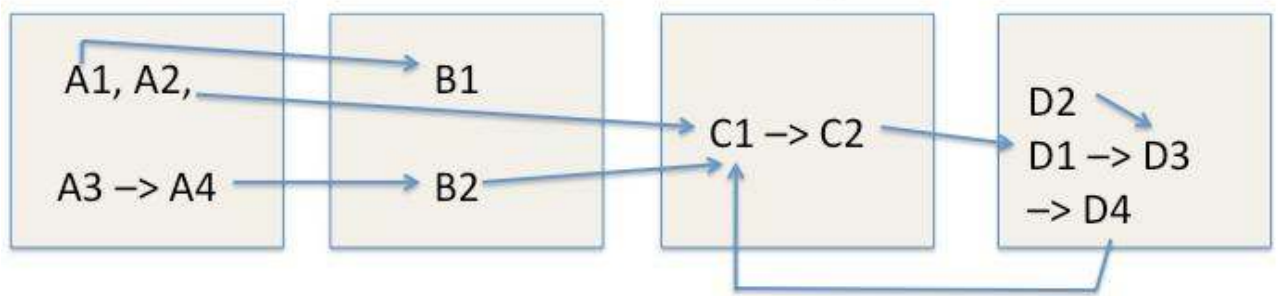

\section{A : CONDITION B : CATASTROPHE C: CONSÉQUENCE D : CONCLUSION}

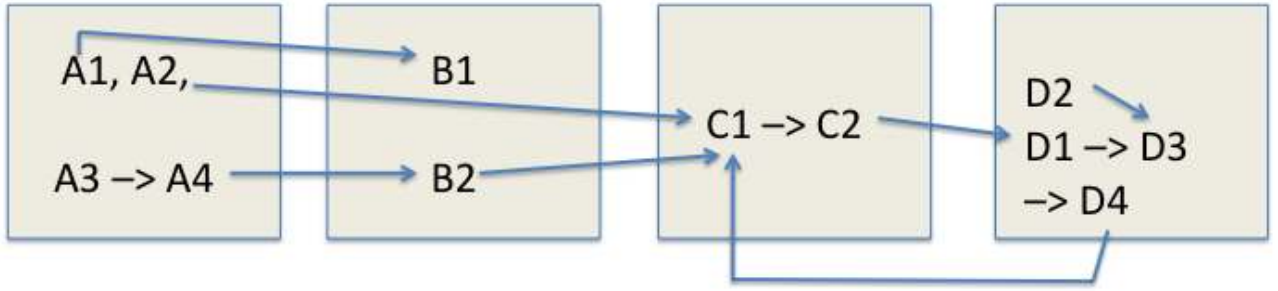

La flèche anaphorique qui va de $\mathrm{D} 4$ aux flèches faisant le lien entre $\mathrm{B}$ et $\mathrm{C}$ semblerait annuler de manière contrefactuelle tout le contenu de C. A3, B2 et D2 sont les événements fatals. Seulement l'espace de conséquence n'en a aucun. Le renvoi vers le passé de D4 attire en retour notre attention sur A2 et, de là, sur la triste fragilité du moment où le rêve de Mathilde s'est réalisé (A1/B1), déclenchant l'humiliation sociale qui s'ensuit (A2 et C, D).

\section{2.}

Dans la nouvelle intitulée Deux amis ${ }^{22}$, nous apprenons quel est le sort de deux citoyens parisiens, M. Morissot et M. Sauvage, un horloger et un mercier, qui avaient l'habitude d'aller à la pêche et de profiter de la douceur du paysage ensemble sur les bords de la Seine. Cependant, le siège de Paris par les Prussiens, pendant la guerre francoprussienne de 1870-1871, les empêche de se rendre sur leur lieu de pêche favori. Ils se rencontrent par hasard dans la rue un matin d'hiver et, après deux verres d'absinthe pris dans des cafés, ils décident de franchir les lignes militaires et d'aller pêcher. Ils obtiennent un laissez-passer du colonel français et se faufilent à travers le territoire occupé jusqu'à la Seine, avec leurs cannes à pêche. Ils peuvent bientôt se laisser aller à la joie d'attraper une abondance de goujons argentés, « cette joie qui vous saisit quand on retrouve un plaisir aimé dont on est privé depuis longtemps ». Mais ils sont bientôt repérés par un groupe de soldats prussiens et conduits sur une petite île de la Seine, l'île Marante. Un officier les informe qu'ils vont être exécutés en tant qu'espions français. Il leur demande quel est le mot de passe et leur promet de les laisser repartir s'ils le lui donnent. Ils sont dans l'incapacité de répondre et se taisent. L'officier pose cérémonieusement la question à chacun d'entre eux, mais il obtient le même silence en réponse. Les deux amis se disent adieu, sont fusillés et jetés dans la rivière. L'officier donne l'ordre, pour finir, qu'on lui prépare la friture de poissons. 
27 L'histoire offre (A) un espace initial, Paris assiégé, puis un second espace, les bords de la Seine, où les protagonistes se laissent aller à la joie de voir leur rêve réalisé (B). Le troisième espace $(\mathrm{C})$ est celui de l'île, où les personnages assument les conséquences de leurs actes, à savoir le fait d'être formellement déclarés espions, même si l'identification n'est que formelle. L'officier accusateur n'a pas à croire en la vérité intrinsèque de cette déclaration, il se contente d'affirmer cela, étant donné le lieu où ils ont été découverts et celui d'où ils viennent. Le statut qu'il leur attribue est le résultat d'un simple calcul stratégique ${ }^{23}$. Ils doivent donc mourir, qu'ils donnent le mot de passe ou non. Il ne semble d'ailleurs pas qu'ils en connaissent un qu'ils pourraient avoir décidé de ne pas révéler ${ }^{24}$. Le quatrième espace (D) est celui (ou inclut celui) du fleuve à la surface duquel flotte leur sang.

28 Essayons maintenant de parvenir à une vue d'ensemble dynamique.

29 A. Paris assiégé.

A1. La force intentionnelle agentive du désir d'aller à la pêche des deux pêcheurs.

A2. La force causale banale des conditions engendrées par la guerre : la famine, la misère. A3. La force causale fatale de la rencontre fortuite des deux amis.

A4. La force intentionnelle agentive de leur prise de contact avec l'officier français et de l'obtention du laissez-passer ${ }^{25}$. Et A3 $\rightarrow$ A4 .

30 B. La pêche sur les bords de la Seine.

B1. La force causale banale de leur bonheur de pêcher de nouveau, qui fait écho à A1.

B2 : La force causale fatale de leur découverte par les soldats prussiens.

31 C. L'île Marante.

C1. La force causale banale de leur identification en tant qu'espions par l'officier prussien. $\mathrm{C} 2$. La force causale banale du verdict : l'exécution. Les règles de la guerre sont suivies. $\mathrm{C} 1 \rightarrow \mathrm{C} 2$.

32 D. Les bords du fleuve sur l'île, lieu de la scène de mort.

D1. La force intentionnelle agentive de la réaction des deux amis à C2.

D2. La force intentionnelle agentive de l'interrogation rituelle des deux " espions » par l'officier. D3. La force intentionnelle agentive de l'adieu que les deux amis échangent. D1 $\rightarrow$ D3.

D4. La force causale banale de l'exécution même, dont les aspects figuratifs renvoient au complexe $\mathrm{A} 2-\mathrm{B} 2$, qui conduit à $\mathrm{C} 1$, c'est-à-dire aux règles d'un temps de guerre. L'annulation des règles humaines de respect et de solidarité (les soldats ne sont pas autorisés à agir selon une éthique ordinaire).

33 Le réseau dynamique des Deux amis est presque comparable ${ }^{26}$, sur le plan formel, à celui de La parure, dans la mesure où les forces sont liées exactement de la même manière, à l'exception du lien A4 $\rightarrow$ B2 dans La parure, absent dans Deux amis. Les forces ne sont pas de la même nature, mais elles conduisent au même renvoi dans le passé, $\mathrm{D} 4$ vers $\mathrm{C} 1$, ce qui, dans les deux cas, donne à l'histoire son sens inquiétant ${ }^{27}$, à savoir celui de la fragilité de la condition humaine engendrée par la division des classes sociales ou par la guerre. La prose de Maupassant offre une richesse de détails allant dans le sens de cette lecture. 
3.

Dans $L a$ ficelle ${ }^{28}$, l'action se situe en Normandie et les paysans, riches ou pauvres, se rendent au marché dans une petite ville ${ }^{29}$. Le personnage principal, maître Hauchecorne, en arrivant en ville, remarque un petit bout de ficelle par terre et le ramasse. C'est une habitude chez lui, car, en bon paysan, il a le sens des économies. C'est alors qu'il se rend compte qu'une personne avec qui il est fâché, maître Malandin, le regarde depuis le seuil de sa maison. Pris de honte d'être vu en train de ramasser un bout de ficelle sans valeur, il le cache et fait semblant de chercher autre chose dans la poussière, puis s'en va, tête basse. Brusquement, à midi, alors que tout le monde est attablé à l'auberge, le tambour retentit et le crieur public annonce la perte d'un portefeuille contenant cinq cents francs et des papiers d'affaires. Un peu plus tard, le brigadier de gendarmerie apparaît et demande à parler à maitre Hauchecorne. Il accompagne le brigadier au bureau du maire. Le maire lui déclare qu'il a été vu en train de ramasser le portefeuille en question. C'est maître Malandin qui l'a vu. Hauchecorne montre alors le bout de ficelle qu'il a trouvé par terre et jure sur "son âme et son salut » qu'il dit la vérité. Le maire ne le croit pas et ajoute qu'on l'a même vu chercher dans la boue si des pièces de monnaie ne s'étaient pas échappées du portefeuille. On fouille Hauchecorne, sans résultat, et on confronte Malandin et Hauchecorne : aucune conclusion ne peut être tirée de cette rencontre. Mais l'histoire se répand. Personne ne croit à la version de Hauchecorne et on se moque de lui. Il insiste, raconte son histoire à tous ceux qu'il rencontre, mais personne ne le croit. Le lendemain, on rapporte le portefeuille à son propriétaire : il avait été trouvé par un valet de ferme illettré qui l'avait donné à son maître, lequel l'avait finalement rendu à son propriétaire. Hauchecorne triomphe et raconte une fois encore son histoire. Il insiste, la répète sur les routes, au cabaret, devant l'église, devant des étrangers. Les gens écoutent en plaisantant, mais ne semblent pas convaincus. Il est même traité de "gros malin » en ville par un autre paysan. Il finit par comprendre, lors d'une conversation à l'auberge, qu'on l'accuse d'avoir volé le portefeuille et de l'avoir fait rapporter par un complice. Il rentre chez lui, honteux et désemparé, conscient du fait qu'il aurait pu faire, en bon Normand roublard, ce dont on l'accuse et qu'il aurait pu, ensuite, s'en vanter. Il s'épuise à répéter son récit et ses justifications, qui deviennent de plus en plus longs et il dépérit. Il s'alite un jour d'hiver et meurt peu après. Dans le délire de son agonie, il continue à clamer son innocence et répète : «Une 'tite ficelle... une 'tite ficelle... t'nez, la voilà, m'sieu le Maire ».

35 La séquence des espaces est articulée de manière claire, bien que (A) inclue géographiquement (B) et que (D) inclue (A) et (C). L'histoire commence sur la route conduisant au marché : c'est l'espace culturel de condition, largement décrit (A). Puis, le lieu catastrophique de la ficelle et de la simulation fatale (B) est présenté. C'est dans le bureau du maire que Hauchecorne révèle la vérité, mais est également rejeté (C). Le reste du récit se sert du paysage dans son entier pour développer les conséquences de cette histoire contre-productive (D). Durant la scène finale d'agonie, le fermier revient mentalement à $\mathrm{C}$.

A. La route vers le marché normand.

A1. La force intentionnelle agentive du désir qui pousse le protagoniste à tirer profit des plus petits avantages ou des plus petites trouvailles.

A2. La force causale banale de la culture paysanne locale, mesquine et malveillante. 
A3. Les circonstances causales banales du jour de marché, à savoir la lecture publique de l'annonce.

B. La scène de la ficelle.

B1. La force causale fatale qui fait que Hauchecorne trouve et ramasse un bout de ficelle. $\mathrm{A} 1 \rightarrow \mathrm{B} 1$.

B2. La force causale fatale qui fait que Malandain, personne malveillante, observe la scène et entraîne Hauchecorne à simuler. B1 $\rightarrow$ B2.

C. Le bureau du maire.

C1. La force causale fatale de l'annonce publique concernant la perte du portefeuille.

C2. La force causale banale de la dénonciation que Malandain fait au maire. B2 $\rightarrow$ C2.

C3. La force causale banale de l'accusation de Hauchecorne par le maire. C2 $\rightarrow$ C3.

D. L'espace de la communauté rurale dans son entier.

D1. La force intentionnelle agentive de la réponse narrative faite par Hauchecorne. C3 $\rightarrow$ D1.

D2. La force causale banale de la réaction des paysans devant sa défense. A2 $\rightarrow \mathrm{D} 2$.

D3. La force causale fatale du sentiment - contrefactuel - de culpabilité qu'éprouve Hauchecorne, sentiment qui contribue probablement à ses excès narratifs, le conduit ensuite à revivre l'échec de sa défense devant le maire et, pour finir, le tue.

Nous voyons, une fois encore, un retour sémiotique allant de l'espace de conclusion au contenu même de l'espace de conséquence. Cette fois-ci, ce retour atteint le personnage, du moins de manière confuse, alors que le lecteur comprend aisément que l'excès de justification est contre-productif. Le sujet est de nouveau coupable, dans un certain sens, d'être là où il est (c'est-à-dire à un endroit où attirer l'attention sur soi ou commettre une bêtise peut tuer), et non ce qu'il est (à savoir innocent). La tragédie est en fait provoquée par l'opposition existant entre ce qu'un honnête partage d'expérience narrative devrait créer comme effet parmi des êtres humains et ce qui se produit en réalité, lorsque l'énonciation hyperbolique de ce récit est perçue comme preuve de sa fausseté. Il s'agit, une fois encore, d'une opposition entre le global et le local, entre une paisible humanité globale et une hostilité et une cruauté locales.

41 Cette analyse se traduit par un réseau dynamique légèrement différent du précédent.

Fig. 3. Le réseau spatio-dynamique de La ficelle

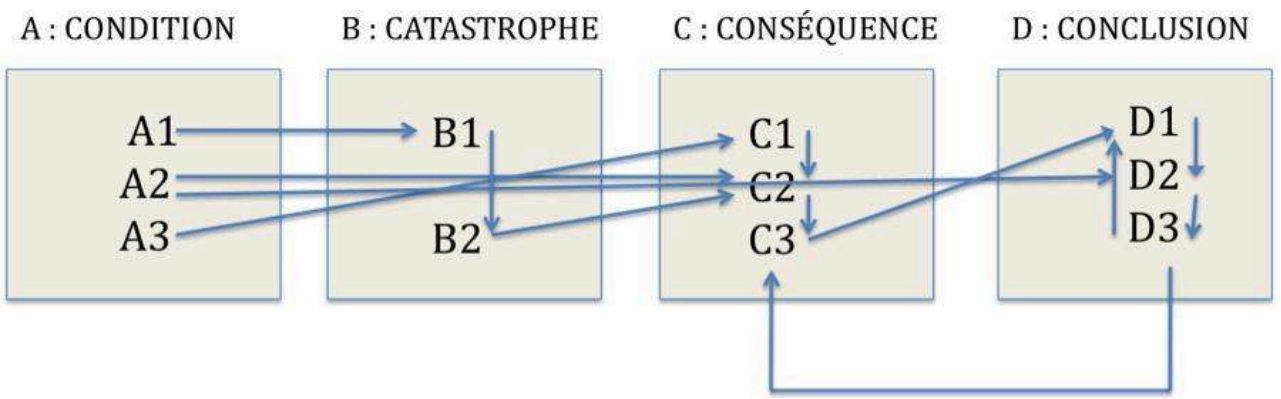

42 Ce réseau, comme nous le voyons, est extrêmement dense, ce qui est peut-être la raison pour laquelle nous sentons combien son sens est attristant, sens dont le lecteur fait l'expérience à travers le retour en arrière $(\mathrm{D} 3 \rightarrow \mathrm{C} 3)$ et la boucle $\mathrm{C}-\mathrm{D}$ qui s'ensuit et qui mène à la mort du personnage.

434.

Changeons maintenant de siècle et examinons une autre histoire remarquable, celle écrite par Jorge Luis Borges, Emma Zunz ${ }^{30}$. Emma est une jeune ouvrière de dix-neuf 
ans, qui travaille dans l'usine de tissus dirigée par Aaron Loewenthal, à Buenos Aires. Un jour d'hiver, elle reçoit une lettre du Brésil, l'informant que son père s'est suicidé. Il avait travaillé pour l'usine en tant que caissier, mais avait été renvoyé pour fraude, six ans plus tôt. Avant de s'exiler, il avait confié à sa fille que le vrai coupable était en fait Loewenthal lui-même, alors gérant de l'usine, devenu par la suite un de ses propriétaires. Emma, profondément affectée, décide au cours de la nuit de se venger du fraudeur, responsable de la mort de son père. Elle attend jusqu'à samedi après-midi, après le travail. Elle se rend au port et trouve un marin étranger, dont le bateau doit appareiller la nuit même. Elle s'offre à lui, comme si elle était une prostituée, alors que l'histoire souligne la profonde horreur qu'elle éprouve pour la sexualité. Elle parvient à son but, mais déchire le billet que son «client » laisse près du lit, de même qu'elle avait déchiré la lettre arrivée du Brésil. Elle regrette toutefois cet acte impie ${ }^{31}$. Ensuite, comme une grève menace l'usine, elle téléphone à Loewenthal et lui demande de le voir en personne, sous prétexte de lui donner des informations sur ses collègues. Elle se rend à l'usine, mais rien ne se passe comme prévu. Elle pénètre dans le bureau de son patron, puis commence à lui parler, de manière hésitante, de ses collègues. Pour lui faire quitter le bureau, elle lui demande un verre d'eau et pendant qu'elle est seule, elle prend le revolver que tout le monde sait se trouver dans le tiroir du bureau. Toutefois, lorsque Loewenthal revient, elle lui tire immédiatement dessus par deux fois, pressée de punir l'outrage auquel elle s'est exposée, plutôt que de venger son père, sans prendre le temps de lui débiter les mots d'accusation qu'elle avait préparés, évoquant le triomphe de la justice de Dieu sur la justice humaine. Durant son agonie, Loewenthal jure violemment, le chien de garde aboie et, finalement, Emma entame son discours : "J'ai vengé mon père et on ne pourra pas me condamner... ", devant un homme déjà mort, qui n'a sans doute rien compris à ce qui lui arrivait. Avant d'appeler la police, elle met «le divan sans dessus-dessous », crée du désordre dans la tenue du mort et lui enlève ses lorgnons tachés de sang. Elle donne par téléphone sa version des faits à la police : elle évoque l'appel que Loewenthal lui aurait passé, sous le prétexte qu'il voulait lui parler de la grève, puis le viol et enfin la légitime défense. On croit son histoire, parce que, comme le narrateur le dit dans son commentaire, son histoire est vraie pour l'essentiel et elle est racontée avec conviction, en ce qui concerne le ton, la honte, la haine et l'outrage ; elle est toutefois fausse pour ce qui est des circonstances, de l'heure et de « un ou deux noms propres».

44 Emma reçoit la lettre déclenchant le processus de vengeance, chez elle. C'est là qu'elle se rappelle le secret que lui a confié son père et qu'elle donne forme à son projet : tout d'abord un espace de condition (A). Puis, elle se trouve dans un bar du port et dans la chambre à l'étage : c'est l'espace de son expérience catastrophique (B). La conséquence, ainsi préparée, est celle de la visite au bureau de Loewenthal (et à son domicile, dans la mesure où il vit «seul à l'étage le plus élevé de l'usine », le lieu du meurtre (C)). Le dernier espace (D) inclut tous les lieux où Emma va répéter l'histoire probable que tout le monde croira.

\section{A. L'appartement d'Emma.}

A1. La force intentionnelle agentive de l'acte frauduleux commis par Loewnthal.

A2. La force causale fatale de la lettre annonçant le suicide paternel. A1 $\rightarrow \mathrm{A} 2$.

A3. La force intentionnelle agentive du plan de vengeance d'Emma. A2 $\rightarrow$ A3. 

échec sur le plan psychologique. La frustration émotionnelle d'Emma (D2) est due à l'horreur de B1, beaucoup plus grave pour elle que son chagrin en A2. Elle est alimentée en outre par l'acte symboliquement inachevé commis en C2. Un des thèmes narratifs les plus «significatifs » en littérature et dans la vie, à savoir la vengeance, la quête d'une justice du " œil pour œil », aboutit à une situation douloureuse, totalement dénuée de sens, et marquée par la banalité et la frustration.

5.

La seconde histoire de Borges, L'autre mort ${ }^{33}$, est clairement fantastique ${ }^{34}$. Le narrateur à la première personne reçoit une lettre de son ami Gannon, l'informant qui est sur le point de lui envoyer la traduction espagnole du poème de R. W. Emerson, The Past, et ajoutant qu'une personne qu'ils connaissaient tous deux venait de mourir d'une congestion pulmonaire : il s'agissait du péon Pedro Damián, originaire de Gualeguay, au nord de l'Argentine, à la frontière uruguayenne, qui avait combattu, quarante ans plus tôt, aux côtés du rebelle conservateur (blanco) uruguayen, Aparicio Saravia, lors de la bataille décisive de Masoller, entre l'armée uruguayenne, dirigée par le président libéral (colorado) et les rebelles. Le caudillo blanco avait été battu et était mort de ses blessures. Au cours du délire fiévreux de son agonie, Damián avait revécu le carnage de Masoller. Il était rentré dans sa province après la bataille et avait passé le reste de sa vie dans sa petite ferme isolée. Le narrateur l'avait rencontré en 1942 et avait tenté de 
parler avec lui, mais c'était un homme taciturne. Gannon avait envoyé au narrateur, autrefois, une photo de Damián, mais cette photo a disparu et le narrateur craindrait de la retrouver (la raison de cette crainte n'est pas précisée). Puis, quelques mois plus tard, à Montevideo, le narrateur rend visite au colonel Tabares, qui a participé à cette même bataille, ce qui intéresse le narrateur, car il prépare une histoire fantastique sur le sujet. Tabares lui livre volontiers de nombreux détails sur les horreurs des batailles de cette guerre civile. Il se trouve qu'il se rappelle Damián, un métis indien dont le souvenir le fait éclater de rire. Le pauvre Damián s'était vanté de son soutien au rebelle blanco, dans les bars, au début des combats, mais s'était comporté lâchement dans la dernière bataille. Le narrateur avait vu un héros possible en Pedro, mais il comprend désormais sa solitude, son silence et ses réserves. C'était un Argentin et il devait être un brave gaucho qui avait été pris dans un combat qui le dépassait. Toutefois, Tabares méprise son manque de courage. Le narrateur rencontre de nouveau Tabares, car l'histoire qu'il veut écrire lui résiste. Il le trouve en compagnie d'un autre homme âgé, le docteur Amaro, qui avait participé lui aussi à la révolution de Saravia, et la conversation tombe, à un moment donné, sur Masoller. Après quelques anecdotes, Amaro mentionne le jeune tondeur nommé Pedro Damián, qui avait participé en première ligne à la charge de cavalerie contre l'ennemi et était mort héroïquement, en criant « ¡Viva Urquiza ${ }^{35}$ !», victime d'une balle en pleine poitrine. Cette bravoure, chez un jeune homme qui n'avait pas vingt ans, avait été étonnante. Le colonel Tabares, en entendant cette histoire, semble perplexe et déclare n'avoir jamais entendu parler de Damián. Un peu plus tard, le narrateur rencontre son ami Gannon dans une librairie anglaise de Buenos Aires et ce dernier nie lui avoir écrit au sujet d'une traduction d'Emerson qu'il aurait entreprise et prétend ne rien connaître d'un tel Damián. Puis, le narrateur reçoit une lettre du colonel qui, brusquement, avait recouvré la mémoire et se souvenait du jeune héros Damián, à la bataille de Masoller. Pendant l'été, le narrateur passe par Gualeguay et espère pouvoir interroger l'homme qui a vu mourir Damián, mais il est mort à l'automne. La ferme de l'ancien péon a disparu. Le narrateur tente de se rappeler les traits de Damián, mais il se rend compte que sa mémoire lui faisait évoquer un célèbre chanteur d'opéra. Pour finir, il essaie de trouver une explication à ces faits étranges. Il aurait pu exister deux Damián ou le narrateur aurait pu rêver le premier. Une amie lui suggère que Damián pourrait effectivement être mort à Masoller, en priant Dieu de le laisser revenir dans sa chère province. Dieu, qui ne peut changer le passé, peut intervenir sur le futur, et il laissa le fantôme de Damián revenir à Gualeguaychú, où son ombre vécut dans la solitude. En prolongeant cette dernière hypothèse, le narrateur trouve la solution après avoir lu le traité De omnipotentia de Pier Damiani (!), consacré au pouvoir de Dieu, que discute Dante dans le Paradis ${ }^{36}$. Damiani soutient, contrairement à d'autres théologiens, que Dieu peut en fait changer le passé. La solution que le narrateur présente alors est que Damián s'est réellement comporté en lâche à Masoller, qu'il a survécu et qu'il a consacré le reste de sa vie à se racheter de sa conduite honteuse, dans sa province, par son dur labeur. Pendant quarante ans, il a espéré et prié que lui soit accordée une seconde chance de prouver son courage, et il l'a finalement obtenu dans le délire de son agonie en 1946. Dieu l'a enfin entendu. Il revit la bataille et il se comporte maintenant en brave. Il meurt au cours de la charge, frappé par une balle en pleine poitrine, au début de 1904. D'une certaine manière, il meurt une première fois en 1946 et une seconde fois en 1904. Il a donc fallu que Dieu corrige le passé. Il a dû changer, par exemple, les souvenirs de tous ceux qui se rappellent Damián et il a dû faire disparaître l'homme qui l'a vu mourir en 1946. Dans la mesure où le 
narrateur l'a lui aussi rencontré, il semblerait qu'il soit en danger ! Mais il échappera bientôt à la menace, du moins l'espère-t-il, vers 1951, car il sera alors convaincu que toute l'histoire n'était qu'une invention engendrée par le poème d'Emerson, par l'analogie entre les noms et par les arguments présentés par Damiani, au cours du processus d'écriture d'une histoire fantastique. Le pauvre Damián a désormais obtenu ce à quoi son cœur aspirait, bien qu'un peu tard, mais peut-être n'y a-t-il pas de bonheur plus grand, conclut le narrateur. Et nous devons lui donner raison, il a bien écrit une histoire fantastique.

52 Ce récit semble insister sur les problèmes de recherche épistémiques d'un narrateur qui est également un écrivain ${ }^{37}$. Il est toutefois facile de constater que l'histoire reconstruite offre les mêmes caractéristiques cognitives que les autres récits analysés dans cet article. Pedro est un jeune paysan argentin vivant à Entre Rios (espace de condition, A). Il se joint aux rebelles uruguayens et combat à Masoller (espace de catastrophe, B). Puis il rentre dans sa province et ressasse, pendant le reste de sa vie, l'issue de la bataille, à la lumière de son comportement moral (espace de conséquence, C). Dieu entend toutefois son désir de rachat au moment de son agonie et lui permet (dans l'espace de conclusion, D) de revenir à Masoller et de rejouer la scène décisive de la bataille : il a, cette fois-ci, la mort glorieuse qu'il a si ardemment désirée.

53 A. Gualeguay, 1904.

A1. La force intentionnelle agentive du désir exprimé par le jeune vantard qu'est Damián, de prouver son courage.

A2. La force causale banale de l'engagement de Damián dans l'armée rebelle de Saravia ${ }^{38}$.

B. Masoller, 1904.

B1. La force causale banale de la violente bataille. $\mathrm{A} 2 \rightarrow \mathrm{B} 1$.

B2. La force causale fatale de la faiblesse psychologique de Damián, le moment de vérité arrivé.

C. Gualeguay, 1904-1946.

C1. La force causale banale de la honte remplissant désormais toute la vie de Damián.

C2. La force intentionnelle agentive de son délire, durant son agonie, au cours de laquelle il revit la bataille finale.

C3. La force intentionnelle magique de la décision divine omnipotente, qui l'autorise à avoir la mort pour laquelle il s'est préparé pendant de longues années et qu'il désire ardemment.

D. Masoller, 1904 revu et corrigé.

D1. La force intentionnelle agentive du comportement brave et de la mort héroïque de Damián, pendant la féroce bataille. D1 révise B2.

D2. La force causale banale des conséquences ontologiques engendrées par le fait que Dieu change le passé : des traces et même des vies humaines sont effacées, celle du narrateur à la première personne inclus.

57 Nous pouvons, une fois encore, résumer ce réseau dynamique dans un déroulement spatio-temporel (fig. 5). 
Fig. 5. Le réseau spatio-dynamique de L'autre mort

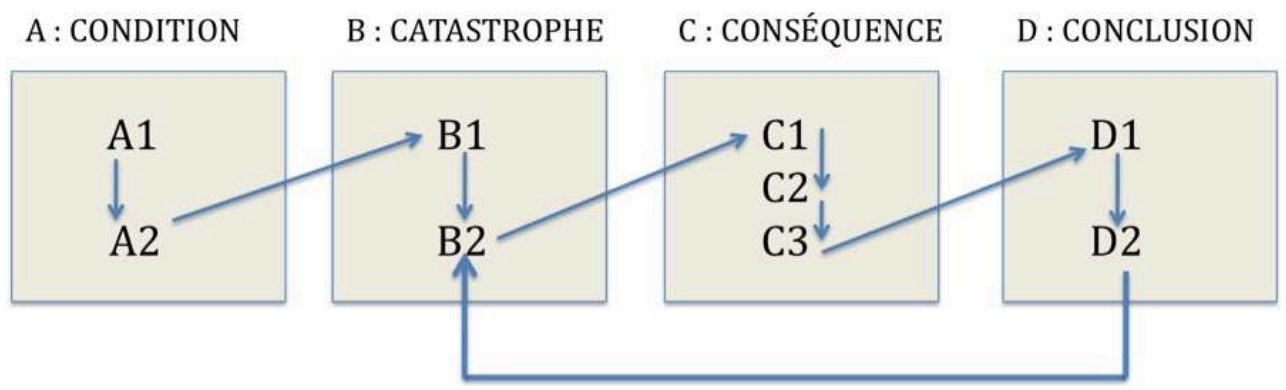

58 La magie ne marche évidemment pas toujours à rebours. Nous savons toutefois que le désir de changer le passé peut être très fort, même dans des esprits contemporains, et c'est, me semble-t-il, une telle disposition intentionnelle que le thème de cette histoire, développé de manière très érudite, exploite. Comme cela a été mentionné précédemment, la cognition humaine est généreuse en termes de dynamique. Elle permet un large éventail de déviations narratives à partir du monde réel banal, comme le savent les philosophes ou les conteurs d'histoires, ou comme ils le découvrent. Borges, quant à lui, a trouvé l'inspiration de son expérience imaginaire dans la théologie médiévale.

\section{6.}

Une très courte histoire ${ }^{39}$ d'Ernest Hemingway se situe pendant la Première Guerre mondiale. Un soldat américain blessé, hospitalisé à Padoue, est soigné par une Italienne, Luz, et tous deux entament une relation amoureuse apparemment sérieuse. Il est opéré et tente de se montrer digne de la femme qu'il aime ${ }^{40}$. Avant que le personnage masculin, qui n'a pas de nom, ne retourne au front, les amoureux pénètrent dans la cathédrale de Padoue, le Dôme, pour prier. Ils auraient aimé se marier, mais ils n'en ont pas le temps et ils n'ont pas non plus d'acte de naissance. La cérémonie n'a pas lieu. La période de séparation est comblée par les lettres ferventes qu'envoie Luz, mais qu'il ne reçoit et ne lit qu'après l'armistice. Il doit alors rentrer aux États-Unis, mais elle refuse de le suivre tant qu'il n'a pas trouvé de travail. Elle lui demande de ne pas voir ses amis (!) et de ne pas boire. Ce sont les conditions qu'elle pose pour le rejoindre plus tard, s'il peut aller la chercher à New York. Ils se disputent à ce sujet dans le train qui les conduit de Padoue à Milan, où ils se séparent. Il quitte l'Italie en passant par Gênes. Luz rentre dans le Nord-est pour travailler à l'hôpital de Pordenone et se sent très seule. Elle rencontre rapidement le major d'un bataillon d'«arditi», les troupes d'intervention spéciale «audacieuses » et glorieuses, et il lui fait l'amour. Elle est très impressionnée par cette relation avec un Italien. Elle écrit alors à Chicago et raconte à son amoureux américain que tout est fini, que leur relation n'avait été qu'une aventure de jeunesse et qu'elle allait bientôt se marier ${ }^{41}$. En réalité, le major ne l'épousera pas. Elle ne recevra aucune réponse de Chicago et le seul "contrat " que le personnage masculin, quant à lui, obtient, est la gonorrhée que lui transmet une vendeuse avec qui il traverse Lincoln Park en taxi. Fin de l'histoire.

Dans cette triste histoire de deux pages, l'espace de condition est l'hôpital de Padoue (A). Je propose que l'espace de catastrophe (B) soit le Dôme, où les amants ne peuvent pas officiellement se marier. C'est là que la symbolisation qui aurait été décisive ne se produit pas $^{42}$. Troisièmement, l'espace de conséquence est incarné par la crise qui a lieu 
dans le train de Milan (C). Enfin, ils cessent de communiquer, se trouvant chacun dans son pays (D) et se laissant absorber par leur misère personnelle, laconiquement évoquée par le narrateur. En termes de logique spatio-temporelle, nous avons ainsi les instances suivantes :

61 A. Padoue. L'hôpital.

A1. La force causale fatale de l'amour.

A2. La force causale banale de la tentative d'amélioration personnelle que fait le personnage masculin pour protéger leur amour.

B. Le Dôme.

B1. La force intentionnelle agentive de la recherche de confirmation symbolique.

B2. La force causale fatale des circonstances empêchant le mariage.

C. Le train allant de Padoue à Milan.

C1. La force intentionnelle agentive des réserves catégoriques de Luz.

C2. La force causale fatale de leur dispute inachevée : le train s'arrête à Milan, le sujet de la dispute n'est pas résolu.

C3. La force causale banale de leur séparation.

D. Chicago et Pordenone.

D1. La force causale fatale de l'infidélité de Luz.

D2. La force causale banale des pratiques sexuelles négligentes de l'ancien soldat.

Dans cette lecture (incluant B2 $\rightarrow \mathrm{C} 1$ ), que je ne prétends pas être la seule possible, le réseau dynamique serait le suivant (fig. 6):

Fig. 6. Le réseau spatio-dynamique d'Une très courte histoire

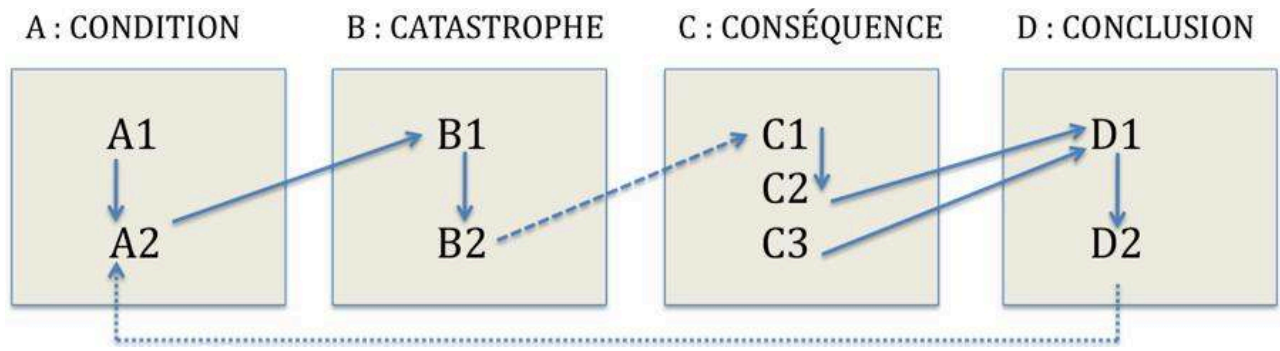

66 Le renvoi de D2 à A2 est une manière rétrospective cruelle de faire écho à A2 et nous permet de relire l'histoire dans une perspective émotionnelle. Sur le plan cognitif, le thème de l'amélioration de soi («tirer vers le haut») ou de l'abandon de tout effort ( (tirer vers le bas ») est, me semble-t-il, une attitude morale universelle liée à l'amour.

\section{Un schéma de dynamique narrative globale}

67 Si nous comparons les structures événementielles présentées ci-dessus, nous pouvons saisir un schéma dynamique trans-spatial. Un sujet désire quelque chose, il l'obtient et réalise son désir, mais il doit payer un prix tristement élevé pour son obtention ou sa réalisation. Le chemin qui conduit au charme (La parure), aux heures de bonheur partagé (Les deux amis), à d'heureuses trouvailles (La ficelle), à la vengeance (Emma Zunz), à l'honneur (L'autre mort) ou simplement à l'amour (Une très courte histoire), passe par la misère, l'humiliation, la souffrance interminable, la mort ou autres sacrifices. Les sujets obtiennent d'une certaine manière ce qu'ils désirent, mais la contrepartie qu'ils ont à 
payer aurait probablement modifié ou peut-être annulé leur désir initial, s'ils avaient eu conscience de ce prix avant de le payer.

En termes de sémantique linguistique, une version spéciale de la dynamique de forces, le schéma de forces et de barrières, utilisé essentiellement pour les analyses de causation, de modalité, et autres phénomènes semio-syntaxiques en grammaire (cf. la note 9, ci-dessus), pourrait servir de représentation diagrammatique de ce motif (fig.7).

Fig. 7. Le schéma dynamique de l'expérience marquante. Contourner la barrière pour atteindre son but et payer le prix :

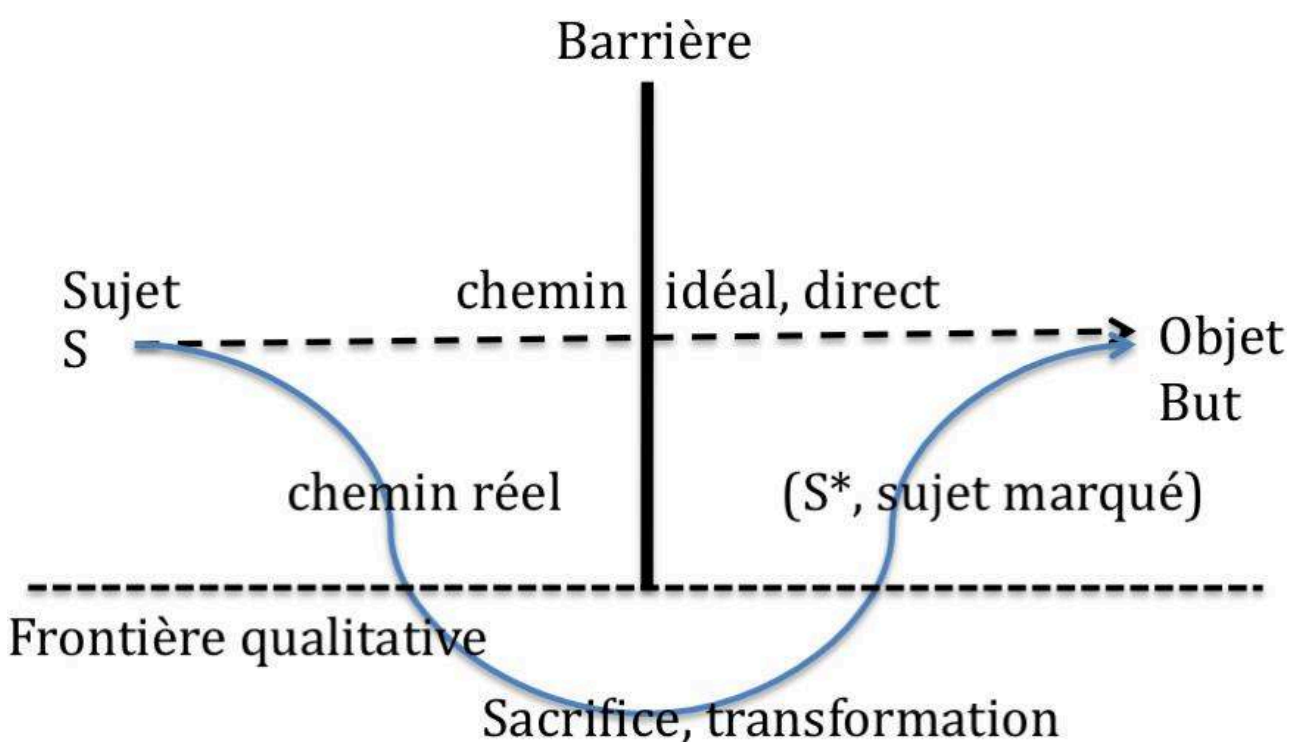

Le sujet doit contourner la barrière qui rend impossible le chemin idéal vers l'objet du désir, et doit ainsi franchir une limite qualitative au-delà de laquelle la vie lève un tribut. Ironiquement, le sujet aura bien atteint sa destination, son objet, son but, mais il sera profondément affecté, marqué par le prix très lourd à payer, sous forme de durée de vie, de dignité, etc ${ }^{43}$.

70 Il existe probablement de nombreuses métahistoires sous-tendant les produits spatiodynamiques diégétiques de l'imagination humaine, mais ce motif sacrificiel est l'un des plus marquants.

\section{Conclusion}

71 Le lecteur aura parcouru les réseaux dynamiques construits dans ce texte et aura eu la possibilité d'évaluer les relations particulières qui ont été présentées et postulées. Les réseaux relationnels sont au cœur de ce qui est significatif dans ces histoires, de leur production de sens, si l'on préfère, dans la mesure où ces réseaux s'impriment dans les esprits des lecteurs et les interrogent par rapport à leurs propres expériences des enchaînements événementiels possibles, et par rapport aux sentiments qu'ils peuvent avoir face à ce qui leur arrive.

72 Dans les nouvelles comme celles que nous avons étudiées, nous pouvons saisir un déroulement narratif élémentaire, une sorte de molécule narrative, dont les atomes sont les espaces dynamiques impliqués. Les forces sont donc les particules de ces atomes, pour filer la métaphore. Dans les créations narratives plus longues, nous 
pouvons supposer qu'apparaissent les réseaux globaux de ces réseaux élémentaires, articulés de manière parallèle ou sérielle. Cette généralisation exige bien entendu un examen empirique.

73 L'analyse dynamique de base est toutefois possible dans la mesure où les forces narratives actives ${ }^{44}$ peuvent être identifiées et localisées de manière spatio-temporelle, dans un format permettant la description et la comparaison. Si la logique dynamique du sens narratif que nous avons ébauchée est stable, lorsque l'on généralise et qu'on évoque des variations culturelles empiriques, elle permettrait alors d'atteindre finalement un niveau d'analyse contribuant tant à la phénoménologie narrative de l'imagination humaine naturelle - et pas seulement de la perception (bien que la perception soit imprégnée de narration ${ }^{45}$ - qu'à la compréhension des cultures humaines comme manières plus ou moins élégantes, complexes, dramatiques, spirituelles ou humoristiques d'exprimer le tissu élaboré des choses que nous faisons et de celles qui semblent tout simplement nous arriver, à nous, à nos dieux, saints, martyrs, héros, collègues, amis et êtres aimés, à savoir à tous ceux à qui nous tenons.

\section{BIBLIOGRAPHY}

Barnatán, Marcos Ricardo, 1981, Jorge Luis Borges, Narraciones. Madrid : Cátedra.

Barthes, Roland, 1977 (1981), « Introduction to the Structural Analysis of Narrative ». Transl.

Stephen Heath. In : Image Music Text. New York : Hill and Wang.

Barthes, Roland, 1968, «L'effet de réel », Communications No. 11, Paris : EHESS.

Baudrillard, Jean, 1983, Les stratégies fatales, Paris : Édition Bernard Grasset, série Figures.

Benveniste, Émile, 1966, «L'homme dans la langue », chap. in Problèmes de linguistique générale. Paris : Gallimard.

Bordwell, David, 2007, Poetics of Cinema. New York : Routledge.

Borges, Jorge Luis, 1980, Prosa Completa, I \& II, Barcelona : Bruguera.

Brandt, Line, 2001, Explosive Blends - From Cognitive Semantics to Literary Analysis. Thesis. Depts. of Philosophy and English. Roskilde University.

Brandt, Per Aage, 2007, « On Consciousness and Semiosis ». In : Cognitive Semiotics, 1, Berne : Peter Lang Verlag.

Brandt, Per Aage, 2004a, Spaces, Domains, and Meaning. Essays in Cognitive Semiotics, European Semiotics Series, 4 , Berne : Peter Lang.

Brandt, Per Aage, 2004b, « Toward a Cognitive Semiotics ». In (ed.) Pierre Fastrez, Recherches en Communication, 19, Dossier Cognitive Semiotics, Leuven : Université Catholique de Louvain.

Brandt, P. Aa., 1995, Morphologies of Meaning. Aarhus : Aarhus University Press. 
Brandt, P. Aa., 1994, « Curiouser and curiouser - A Brief Analysis of Alice in Wonderland », in (ed.) Carla Marello, Semiotics and Linguistics in Alice's Worlds, Ed. Walter de Gruyter, Series Research in Text Theory/Untersuchungen zur Texttheorie, Berlin, New York.

Brandt, P. Aa., 1992, La charpente modale du sens. Pour une sémio-linguistique morphogénétique et dynamique. Aarhus, Amsterdam : Aarhus University Press \& John Benjamins.

Brandt, P. Aa., 1990, « The Dynamics of Modality : a Catastrophe Analysis », RSSI (Recherche Sémiotique/Semiotic Inquiry), Vol. 9, 1-2-3, Université du Québec à Montréal.

Brandt, P. Aa., 1983, Sandheden, sætningen og døden. Semiotiske aspekter af kulturanalysen [Truth, Sentence, Semiotic Aspects of Cultural Analysis]. Copenhagen : Basilisk.

Cervoni, Jean, 1987, L'énonciation. Paris : PUF, série Linguistique nouvelle.

Colm Hogan, Patrick, 2003, Cognitive Science, Literature, and the Arts. New York, London : Routledge. Coquet, Jean-Claude, 1997, La quête du sens. Le langage en question. Paris : PUF.

Evans, Vyvyan, 2006, The Structure of Time : Language, Meaning, and Temporal Cognition. Amsterdam, Philadelphia : John Benjamins, series Human Cognitive Processing.

Fludernik, Monika, 2005 (1996), Towards a « Natural' Narratology ». London, New York : Taylor \& Francis.

Gavins, Joanna, 2007, Text World Theory. An Introduction. Edinburgh : Edinburgh University Press. Genette, Gérard, 1980 (from 1972, Figures III), Narrative Discourse. Ithaca : Cornell University Press.

Genette, Gérard, 1988, Narrative Discourse Revisited. Transl. Jane E. Lewin. Ithaca : Cornell. U. P. Geeraerts, Dirk and Cuyckens, Hubert, 2007, The Oxford Handbook of Cognitive Linguistics. Oxford : Oxford University Press.

Greimas, Algirdas Julien, 1976, Maupassant. La sémiotique du texte. Exercices pratiques. Paris : Éditions du Seuil.

Greimas, Algirdas Julien, 1966, Sémantique structurale. Recherche de méthode. Paris : Larousse.

Hemingway, Ernest, 1925, In Our Time, New York : Boni \& Liveright.

Herman, David, 2004, Story Logic : Problems and Possibilities of Narrative. Lincoln : University of Nebraska Press.

Herman, David, 2000, « Narratology as a cognitive science ». In Image \& Narrative, Vol. 1 : Cognitive Narratology, www.imageandnarrative.be

Jakobson, Roman, 1960, «Closing Statements : Linguistics and Poetics », in (ed.) Th. Sebeok, Style in Language, Cambridge, Mass. : MIT Press.

Maupassant, Guy de, 1974, Contes et nouvelles, I, ed. Louis Forestier. Paris : Gallimard, Bibliothèque de la Pléiade.

Stafford, Barbara Maria, 2007, Echo Objects : The Cognitive Work of Images. Chicago : University of Chicago Press.

Sternberg, Meir, 1993, Expositional Modes and Temporal Ordering in Fiction. Bloomington : Indiana University Press.

Sweetser, Eve, 1990, From Etymology to Pragmatics. Cambridge : Cambridge University Press.

Talmy, Leonard, 2000, Toward a Cognitive Semantics. I \& II. Cambridge, Mass. : MIT Press. 
Todorov, Tzvetan, 1969, Grammaire du Décaméron. The Hague, Paris : Mouton

Todorov, Tzvetan, 1970, Introduction à la littérature fantastique, Paris : Seuil.

Thom, René, 1980, Modèles mathématiques de la morphogenèse, Paris : Christian Bourgois Éditeur.

Tulving, Enver, 1983, Elements of Episodic Memory. Oxford : Clarendon Press.

Turner, Mark, 1996, The Literary Mind. The Origins of Thought and Language. New York : Oxford University Press.

Zacks, Jeffrey M. and Magliano, Joseph P., (2009, in print), « Film, Narrative, and Cognitive Neuroscience ». In (ed.) Melcher, D.P. and Bacci, F., Art and the Senses. New York : Oxford University Press.

\section{NOTES}

1. Au sens du discours qui, respectivement, raconte, montre et démontre. Benveniste (1966) a étudié les différences structurelles du récit et du discours, en distinguant, essentiellement, le mode narratif des autres modes.

2. L'espace référentiel d'une description peut être strictement conceptuel ou notionnel et, dans ce cas, la présentation de la distribution notionnelle peut, bien entendu, être temporelle. On peut penser aux recettes de cuisine, par exemple. Ce n'est pas parce que les actions prescrites ne doivent pas être réalisées simultanément que ces textes seraient narratifs.

3. La fictivité de la fiction n'est pas notre problème dans l'analyse de la logique narrative événementielle; cette caractéristique des histoires bien composées vient probablement de leur structure fermée, de l'impression d'achèvement qu'elles donnent. Elles nous font penser : se non è vero, è ben trovato! Quoi qu'il en soit, la fictivité est un phénomène qui constitue un défi sémiotique et cognitif. Je pense que Roman Jakobson (1960) était sur la bonne voie, lorsqu'il a proposé son concept de fonction poétique fondée sur l'auto-référence du «message »: si une histoire offre une structure à ce point marquante et captivante que sa vérité importe peu, elle sera alors artistiquement racontée hors du contexte des choses vraies et transmise par exemple en vers ou en prose, afin d'être appréciée par des publics qui ne connaissent pas les circonstances référentielles de son origine. On pourrait dire que son « artisterie » en fait un « même » (Richard Dawkins, Le gène égoïste, 1976). Une autre théorie voit la fictivité comme l'introduction, dans le discours, d'un narrateur "fictif», artificiel, c'est-à-dire d'un rôle énonciatif et théâtral qui détermine tout ce qui est dans son champ comme irréel, puisqu'il est lui-même irréel. Les deux théories peuvent converger.

4. Turner (1996) emploie le terme d' « histoire » dans le sens plus large d'un état, d'un événement ou d'une situation quelconque impliquant un sujet; son emploi élimine le sens dramatique du terme.

5. Quand le narrateur est incarné dans une instance primaire subjective, nous avons souvent l'impression que le narrateur «olympien" propre à la narration à la troisième personne (tel une voix extérieure incarnée) disparaît : nous avons alors affaire à une narration à la première personne. Mais l'on peut également entendre, à bon droit, cette dernière comme un récit discrètement polyphonique: "Il faisait un beau jour d'été à Paris. J'étais assis sur un banc dans le parc, profitant du soleil et lisant mon journal, lorsque, brusquement... ». La première phrase relève du style « olympien ", alors que la seconde a un narrateur incarné. La voix incarnée ne supprime pas nécessairement la voix désincarnée, ce qui donne la possibilité d'un duo ou, en général, d'une pluralité de voix, parmi lesquelles l'une reste désincarnée. 
6. Cette idée n'est, en aucune manière, banale ou évidente. Greimas (1966) a proposé de situer sa dynamique narrative actantielle à un niveau intermédiaire entre le niveau sémiotique profond de pure « logique » oppositionnelle et le niveau superficiel du discours chargé d'énonciation et de rhétorique, dans une théorie sémantique "générative » du sens. Colm Hogan (2003) commence un chapitre en affirmant que: "Une distinction centrale en narratologie est à établir entre l'histoire et le discours, ce qui arrive et la présentation de ce qui arrive ». Ce qui arrive est ce que j'appelle l'histoire en soi.

7. Je ne prétends pas, bien évidemment, que la narratologie devrait se borner à l'étude de la structure de l'histoire, mais uniquement que la différence entre les deux principaux aspects des récits devrait être reconnue. Alors que l'étude de la narration, des styles du narrateur, des points de vue, des stratégies narratives, etc., c'est-à-dire l'étude de la rhétorique des récits, est au centre de la recherche littéraire depuis un siècle, l'aspect propre à l'" histoire » a été négligé à tel point qu'il est maintenant courant de croire qu'il n'existe pas de structure de l'histoire dans les textes narratifs, seulement un processus "narrant " et un processus correspondant de réception en cours. Cette vision des choses convient au style de pensée empirique de certains chercheurs philosophiquement partiaux - David Bordwell (2008) pourrait en être un exemple récent. C'est la raison pour laquelle un des buts premiers de cet article est de montrer qu'il existe vraiment une structure de l'histoire, indépendante des styles du narrateur, comme en linguistique, où il est en fait possible de distinguer un énoncé de son énonciation (Benveniste 1966, Cervoni 1987). Pour être précis : le fait « qu'il y ait une histoire » ne signifie pas qu'elle existe sur un écran, une feuille de papier, bref, matériellement, de même que la sémantique d'une phrase ne vit pas dans la manifestation des sons de l'expression orale. Mais où vit-elle alors? Dans le contenu cognitif des esprits qui partagent sémiotiquement le sens d'une phrase dans la communication. Les esprits peuvent-ils vraiment partager un sens? Oui. Comment? En suivant les instructions sémiotiques du signe échangé. Qu'est-ce qu'un signe ? C'est une longue histoire... On me demande ce qui se passe si l'histoire d'un narrateur réaliste est reprise par un narrateur fantastique. Une histoire peut, bien entendu, subir des transformations telles qu'elle devient fantastique, par exemple, alors qu'elle a été réaliste (ou l'inverse), mais cela ne signifie pas qu'elle change de narrateur, mais d'auteur. Auteur et narrateur doivent absolument être séparés. Une biographie réaliste peut ainsi devenir une hagiographie (fantastique).

8. Quand un changement est-il critique ? Le caractère critique des choses, dans ce contexte, peut être défini comme le fait d'avoir des effets entraînant le surgissement d'autres effets, pas nécessairement immédiats, mais se produisant dans le continuum spatio-temporel de l'histoire concernée. Un changement dynamique est «critique» s'il provoque un autre changement dynamique quelque part. Certaines idées analytiques de base concernant le caractère critique des faits et les rôles de causalité dans la narration en termes de fusion (blending) d'espaces mentaux sont présentés dans Brandt 2004a, chap. 4.

9. On connaît les schémas de dynamique de forces dans la grammaire cognitive (Sweetser 1990, Brandt 1990, Talmy 2000, Geeraerts et Cuyckens 2007), mais la sémantique de la causalité et de la modalité, fondée sur la dynamique de forces, dont on sait désormais qu'elle s'exprime lexicalement et syntaxiquement dans les phrases, n'a pas encore été étudiée systématiquement dans le cadre de récits entiers. La dynamique de forces est toutefois une composante de tous les schémas de trajectoires (source-chemin-but) et de toutes les relations médiatisées entre le sujet et l'objet. Le rôle des formes et des forces dans la perception humaine explique la coopération de la cognition spatiale et de la cognition temporelle dans l'expérience immédiate. La cognition narrative s'appuie sur cette coopération dans la perception et la réactive dans l'imagination humaine, en particulier dans l'anticipation et la préparation des actes et des réactions. Toutes les expériences du temps sont dynamiques. Voir Evans 2006. 
10. J'entends par là tout simplement que, lorsque quelque chose nous arrive, nous pensons «c'était surtout à cause de X...». C'est-à-dire que X représente en quelque sorte une force provoquant ce qui est arrivé.

11. Notre mémoire épisodique (Tulving 1983) est formée d'expériences de ce genre. Cela ne signifie pas que les êtres humains perçoivent leur vie comme un récit continu (Stafford, 2007, proteste contre un tel pan-narrativisme existentiel), mais cela signifie que la logique événementielle narrative est directement liée aux processus structurant le caractère épisodique de la mémoire humaine, et que c'est ainsi un phénomène qui relève de notre réalité neuropsychologique. Zachs et Magliano (2009) résument de manière très utile un large éventail d'études portant sur la cognition narrative au cinéma et offrent une perspective neuroscientifique actualisée.

12. Ces forces fatales - cf. Baudrillard, 1983, un des essais les plus inspirants et stimulants que j'ai lus sur les actes humains et leurs motivations - sont vécues comme "plus contingentes que la contingence ». Elles sont si fortuites qu'elles semblent exprimer une détermination, destination ou destinée sous-jacentes ou globales. Par exemple, une personne gravement malade s'adresse au Destin, au Fatum: "Pourquoi moi ?». Le fatal devient une fascination dans la manie du jeu. Devrions-nous rejeter ce genre de phénomènes dans la narratologie ? Ils sont irrationnels, dans la mesure où la cognition humaine est (en partie) irrationnelle, quoique sémiotiquement rationnelle, c'est-à-dire qu'elle a un sens.

13. Comme je l'ai mentionné ci-dessus, ma tentative de classification des forces ne contient jusqu'à maintenant que les catégories suivantes : intentionnelle (agentive versus magique) versus causale (banale versus fatale). Cela peut s'avérer trop simple. Toutefois, cette classification semble suffire dans les analyses que je propose ci-dessous.

14. L'idée d'une densité dynamique variable par «addition » ou par « soustraction » de forces est due à Line Brandt (2001). L'idée d'un point de départ réaliste des variations et des dérivations va directement à l'encontre de la suggestion de Roland Barthes dans son célèbre article « L'effet de réel » (Barthes, 1968). Pour lui, il s'agit de comprendre le genre réaliste comme le résultat d'un assemblage d'« effets » descriptifs, obtenus en ajoutant des détails arbitraires, et de voir ainsi le réalisme comme une construction particulièrement artificielle.

15. Le terme français « merveilleux » renvoie à l'univers des contes de fées (cf. Todorov, 1970).

16. Voir « Curioser and curioser », Brandt (1994 et 1995).

17. Maupassant (1978 : 1198). Le Gaulois 1884.

18. Cette structure rappelle évidemment celle du conte de fées Cendrillon (Aarne-Thompson, type $510 \mathrm{~A})$.

19. J'entends par "espaces" soit des lieux géographiques concrets, soit des lieux d'habitation socio-géographiques moins concrets. Les termes proposés ci-dessus les désignant sont génériques. Le terme de "catastrophe » renvoie à la dite "théorie des catastrophes " de René Thom (Thom, 1980; Brandt, 1992): une catastrophe est un changement significatif, dynamiquement provoqué par une dynamique de forces, dans une situation de référence.

20. D4 est fatal au personnage principal sur le plan mental, puisqu'il lui dit que toutes ses souffrances ont été inutiles. Ce que vous ne savez pas et ce que vous apprenez par hasard ou par coïncidence, au lieu de ne pas le savoir et d'être plus heureux dans votre misère, est fatal, mais ne change rien à la logique événementielle, si ce n'est dans la réflexion finale du personnage et du lecteur faisant preuve d'empathie. Cela conduit certainement à un événement épistémique, à savoir la compréhension qu'une grande partie de notre vie est le résultat des conséquences engendrés par de petits malentendus, et dont le prix à payer est élevé.

21. La série d'espaces conceptuels - directement traduite en termes de lieux physiques d'extension variable - est canonique, dirais-je, et elle peut ainsi être comparée aux modèles rhétoriques classiques (exposition, péripétie, climax, dénouement, cf. l'analyse des tragédies grecques que propose Gustav Freytag, 1863 et, dans la poétique moderne, Sternberg, 1993), mais 
cette série est indépendante de la rhétorique. Elle est intrinsèquement le produit d'une dynamique événementielle. Elle ne décrit pas (comme le «climax ») les sentiments attendus de l'auditeur-lecteur, mais le déroulement dans l'espace et dans le temps de la logique événementielle impliquée dans une histoire.

22. Maupassant (1974 : 732). Gil Blas, 1883.

23. Si les deux amis étaient autorisés à rentrer à Paris, ils pourraient raconter à l'armée française où se cachent ses ennemis. Ils seraient potentiellement et rétrospectivement des éclaireurs et par conséquent des espions. Pêcheurs et espions, tout dépend du point de vue. Ils se voient, quant à eux, historiquement, comme des pêcheurs, nous le savons. Mais pour l'officier prussien, qui ne comprend que le jeu de la guerre, ils jouent un autre rôle, de manière aussi véridique que le premier rôle qui était le leur.

24. Greimas (1976), dans son livre (résumé dans Brandt, 1983, chapitre 6, où Deux amis et La ficelle sont réanalysés) entièrement consacré à l'analyse de cette nouvelle, suppose qu'ils ont un mot de passe et pas seulement un laissez-passer oral ou écrit. C'est pourquoi, selon Greimas, ils accomplissent un acte héroïque, en se taisant pendant l'interrogatoire et en s'opposant à l'officier «stalinien », au lieu de sauver leur vie, ce qui aurait été possible et aurait pu se produire s'ils avaient révélé le mot de passe. Jean-Claude Coquet (1997 : 25-26) pense que cette analyse de la situation n'est pas correcte et je partage sa conclusion : ils n'auraient pas pu sauver leur vie. Ils n'ont pas de mot de passe et même s'ils en avaient eu un, il ne leur aurait servi à rien, car l'officier prussien ne peut pas les laisser partir. Ce ne sont pas d'héroïques résistants, mais de simples êtres humains pris dans une logique de lieu et de pouvoir et qui se sont trouvés à un endroit où ils n'auraient pas dû être. C'est la guerre : ils sont désormais des espions (potentiels), étant donné l'endroit où ils se trouvent.

25. «Ils se remirent en marche, munis d'un laissez-passer ». Il s'agit très certainement d'un papier portant leur nom, qui leur permet de franchir les lignes françaises, et non d'un mot d'ordre appris par cœur.

26. Dans cette histoire, l'événement de clôture D4 est banal, alors qu'il était fatal dans l'histoire précédente. L'exécution des pêcheurs est banale dans la mesure où elle suit les règles élémentaires du jeu de la guerre, et c'est précisément ce qui donne à ce texte un sens tragique. Si vous vous trouvez au mauvais endroit, aucune auto-interprétation intentionnelle ne peut vous sauver d'une allo-interprétation correspondant à ce lieu.

27. Dans la mesure où le modèle spatio-dynamique s'avère présenter un réseau structural quasi identique dans ces deux nouvelles, notre figure 2 servira pour toutes les deux.

28. Maupassant (1974 : 1080). Le Gaulois, 1883. L'éditeur offre ce commentaire (p. 1065) : « Un des plus célèbres contes de Maupassant n'évoque pas seulement la terre normande, avec ses coutumes et ses hommes, il montre aussi comment un homme devient aussi prisonnier de sa propre mésaventure ». Il y a là quelque chose de kafkaïen et de labyrinthique, au point que le conte ne peut se refermer que sur lui-même pour recommencer à nouveau: «Les plaisants maintenant lui faisaient conter "la ficelle". Le conte ramené à son statut de pur objet fonctionne comme un piège de la conscience ».

29. «Tout cela sentait l'étable, le lait et le fumier, le foin et la sueur, dégageait cette saveur aigre, affreuse, humaine et bestiale, particulière aux gens des champs ». C'est le naturalisme résumé en une phrase : les êtres humains sont toujours des animaux.

30. El aleph, 1949. Un éditeur et critique de Borges, Marcos Ricardo Barnatán (1981:149) écrit que ce texte, un des rares, chez Borges, à contenir une évocation sexuelle, est en fait une histoire double, une histoire de vengeance et une histoire de répétition de la souffrance de l'être aimé vengé.

31. Le texte dit: "Détruire l'argent est impie, de même que jeter le pain; son geste à peine achevé, Emma se repentit. Un acte d'orgueil et en ce jour... » (Traduction de l'espagnol par René L.-F. Duran, L'Aleph, Gallimard, L'imaginaire, 1967, p. 82). Ma lecture doit accepter le 
commentaire du narrateur sur le fait de déchirer des billets de banque : c'est un acte symbolique, qui pourrait avoir des conséquences, en particulier un jour pareil... Si cet acte a des conséquences magiques, il s'agit alors d'une histoire fantastique, sinon, elle reste dans un registre de réalisme psychologique.

32. Le sentiment magique intentionnel perdure, à savoir que B2 peut avoir provoqué $\mathrm{C} 2$, précisément, si c'est le cas, par magie. Dans ce cas (voir note 22), une fatalité en engendre une autre, puisque la première est en outre à considérer comme négativement intentionnelle par magie - elle touche à un tabou, à une instance intentionnelle (surnaturelle), protégeant des objets symboliques (l'argent, le pain...) de la destruction. En raison de $\mathrm{B} 2 \rightarrow \mathrm{C} 2$, cette histoire pourrait donc être qualifiée de fantastique.

33. El aleph, 1949.

34. Elle est aussi très compliquée et le fait de la discuter dans ce contexte est un défi. Sa part argumentative est écrasante, comparée à sa référence narrative. Il s'agit d'une histoire qui parle d'une histoire et c'est cette dernière qui est au centre de mon analyse.

35. Ce cri est anachronique, puisque Urquiza était le général argentin qui avait lutté contre le dictateur Rosas à la bataille de Caseros, en 1852, et avait entraîné sa chute.

36. Pier ou Pietro Damiani, 1007 - 1072, théologien et saint, est placé dans le septième ciel du Paradis par Dante, XXI, 112 - 126.

37. L'effet des mystères épistémiques de la narration sur le lecteur est certes essentiel au pouvoir esthétique de ce texte. Lire est un processus, et c'est dans la réaction émotionnelle attendue durant ce processus que se trouve principalement l'artifice d'un récit de ce genre et, en général, des histoires fantastiques, qui misent bien entendu sur l'ambiguïté. L'histoire doit toutefois produire un réseau intelligible de relations dynamiques causales à reconstruire, durant le processus de lecture, afin d'avoir un sens, même si cela est (au départ) un mystère, (et un paradoxe final). Si le lecteur ne se demande pas «ce qui s'est réellement passé ", il ne sera pas sensible au mystère.

38. Le texte souligne suffisamment que le motif de sa participation à la bataille n'est pas vraiment la politique uruguayenne de l'époque (Urquiza !).

39. In Our Time, 1925, 1930.

40. "Quand on l'endormit, il se concentra pour ne rien dire au moment ridicule où on raconte des histoires ». Le lecteur se demande quoi penser de cela.

41. «Elle l'aimait toujours, mais elle s'était rendu compte que ça n'avait été qu'une amourette. Elle espérait qu'il ferait une brillante carrière et lui faisait entière confiance. Elle savait que c'était très bien ainsi ». L'ironie mordante du narrateur est évidente dans de telles phrases.

42. «Ils se considéraient eux-mêmes comme mariés, mais ils voulaient que tout le monde le sache, et être plus sûrs de ne pas se perdre ». Le sentiment en tant que tel est insuffisant, il doit être accompagné du rituel symbolique.

43. Le changement se produisant quand le chemin réel (non le chemin idéal) franchit une limite qualitative affecte la situation du sujet de multiples manières et détermine souvent d'autres récits impliquant le sujet, comme nous le voyons dans La parure. Le motif faustien, le fait de vendre son âme au diable, en est un autre exemple frappant. Les chaînons narratifs causaux, tels que ceux que nous avons étudiés, correspondront souvent à toute une variété de scénarios de dynamiques de forces reliés entre eux. Comment se fait-il que nous saisissons intuitivement et avec autant de facilité ces connexions complexes dans le déroulement d'une histoire que nous lisons ? Je pense que la réponse est la même que celle que nous pouvons apporter au problème de notre compréhension automatique rapide de la grammaire et du sens des phrases : nous utilisons des formats cognitifs par défaut.

44. Le terme de forces, je le répète, désigne toutes sortes d'instances causales auxquelles les récits renvoient. Nous devrions être capables de trouver, en dernière instance, un large ensemble de types de forces en littérature; et ensuite, nous devrions, pour l'intérêt de la psychologie 
cognitive, les inclure dans l'inventaire des causes auxquelles l'esprit humain peut recourir dans sa pensée explicative. Il est déjà évident que cet ensemble n'est pas réduit à la causalité physique banale, mais qu'il inclut au moins trois autres types d'emblée, c'est-à-dire sans transfert métaphorique - à savoir, les types que j'ai évoqués ici. L'esprit n'est pas physicaliste. Il serait plus précis de le voir comme spontanément "surréaliste ", au sens large du terme. C'est pourquoi une «naturalisation » des récits (Fludernik, 2005 : 313), construisant « la narrativité sur la base des paramètres cognitifs du monde réel (traité avec souplesse)» devra être suffisamment souple pour comprendre que l'expérience humaine incarnée authentique est intrinsèquement remplie de merveilles magiques, d'humour (ou de tristesse) absurde ou du moins grotesque.

45. La conscience animale est radicalement narrative, dans le sens où elle offre au sujet un espace-temps épisodique plein d'adversaires, d'obstacles, d'alliés potentiels, d'objets de valeur, et de circonstances précaires (Brandt, 2007).

\section{ABSTRACTS}

The aim of a cognitive narratology, as I see it, is to develop the literary and generally semiotic study of narratives through cognitive modeling, and to develop cognitive studies of mind and meaning by integrating insights from literary scholarship. In this article, I first examine the concept of narrative discourse (versus descriptive and argumentative discourse); second, I discuss the principles for distinguishing narrative subgenres (realistic, fantastic, marvelous, grotesque, absurd stories); and third, I propose a model of the constitutive architecture of narrative meaning as manifested by " good stories ", stories that make sense by conveying a view of the human condition.

In order to develop and test the model, I analyze a selection of acknowledged literary masterpieces : three short stories by Guy de Maupassant (Deux amis, La ficelle, La parure), two by Jorge Luis Borges (Emma Zunz, La otra muerte - The other death), one by Ernest Hemingway (A very short story). Through these analyses, including succinct literary interpretations of the texts, a new view of narrative dynamics is outlined. Agents operate in spaces that have specific dynamic properties, in that they display characteristic forces determining acts and events. There is, I postulate, a canonical set of narrative spaces, each encompassing and contributing a significant part of the meaning of a story. The model distinguishes four such spaces, which are typically also staged as distinct locations; an initial conditioning space, a catastrophic space, a consequence space, and a conclusion space.

Forces are described as causal or intentional. The causal forces are either trivial (habitual, regular, default, whether physical or social) or «fatal» (special, contingent, singular). The intentional forces are agentive (volitive and located in agents) or magical (supernatural and nonagentive but still volitive). The scenario-framing, dynamically invested spaces are linked in a default diegetic order allowing forces to fire forwards and backwards, which explains how stories progress and end, and in particular, eventually, how they can mean what we report them to mean. Interpretation and interpretability depend on the dynamic « logic » of this spatial diegesis, more than on reader identifications in a story, or on ideologies ascribed to the narrator.

Le but de la narratologie cognitive, tel que je le vois, est de développer l'étude littéraire et en général sémiotique des récits à partir d'une modélisation cognitive. Il est également de développer les études cognitives de l'esprit et du sens en intégrant l'apport de la critique 
littéraire existante. J'examine tout d'abord, dans cet article, le concept de discours narratif (versus les discours descriptif et argumentatif); ensuite, je discute les principes permettant de distinguer les sous-genres narratifs (histoires réalistes, fantastiques, merveilleuses, grotesques, absurdes) ; enfin, je propose un modèle d'architecture constitutive du sens narratif, tel qu'il se manifeste dans les «bonnes histoires", histoires qui ont un sens, parce qu'elles expriment une vision de la condition humaine.

Afin de développer et de tester le modèle, j'analyse une sélection de textes littéraires considérés comme des chefs-d'œuvre: trois nouvelles de Guy de Maupassant (Deux amis, La ficelle et La parure), deux de Jorge Luis Borges (Emma Zunz et La otra muerte - L'autre mort) et, pour finir une d'Ernest Hemingway (A very short story - Une très courte histoire). À travers ces analyses, incluant certaines interprétations littéraires de ces textes, une nouvelle vision des dynamiques narratives se dessine. Des agents opèrent dans des espaces présentant des propriétés dynamiques particulières, dans la mesure où ces dernières déploient des forces caractéristiques, déterminant des actes et des faits. Je postule qu'il existe un ensemble canonique d'espaces narratifs, chacun d'eux impliquant une part importante du sens de l'histoire et y contribuant. Ce modèle distingue quatre espaces, qui sont également mis en scène comme autant de lieux distincts : un espace initial de condition, un espace de catastrophe, un espace de conséquence et un espace de conclusion.

Les forces sont décrites comme causales ou intentionnelles. Les forces causales sont soit banales (habituelles, ordinaires, soit physiques soit sociales) ou "fatales" (particulières, contingentes, exceptionnelles). Les forces intentionnelles sont agentives (volitives et incarnées dans des agents) ou magiques (surnaturelles et non-agentives, mais toujours volitives). Les espaces dynamiquement investis, qui encadrent les situations, sont liés par un ordre diégétique canonique, permettant aux forces d'avoir des effets prospectifs et rétroactifs. Cela explique le déroulement des histoires, leur fin et le sens que nous leur assignons. Interprétation et interprétabilité dépendent de la "logique » dynamique de cette diégèse spatiale, plus que de l'identification du lecteur avec les personnages d'une histoire ou de l'idéologie attribuée au narrateur.

\section{INDEX}

Mots-clés: narration, cognition, causalité, dynamiques de forces, espaces, Maupassant, Borges, Hemingway

Keywords: narrative, cognition, causation, force dynamics, spaces, Maupassant, Borges, Hemingway

\section{AUTHOR}

PER AAGE BRANDT

Case Western Reserve University 